\title{
ELASTOMERIC IMPRESSION AS A DIAGNOSTIC METHOD OF CAVITATION IN PROXIMAL DENTIN CARIES IN PRIMARY MOLARS
}

\author{
Adriela Azevedo Souza MARIATH ${ }^{1}$, Ana Eliza Lemes BRESSANI ${ }^{1}$, Fernando Borba de ARAUJO²
}

1- MsC, Department of Pediatric Dentistry, School of Dentistry, Federal University of Rio Grande do Sul, Porto Alegre, RS, Brazil.
2- MsC, PhD, Department of Pediatric Dentistry, School of Dentistry, Federal University of Rio Grande do Sul, Porto Alegre, RS, Brazil.

Corresponding address: Fernando Borba de Araujo - Rua Ramiro Barcelos 2492 - Bom Fim - 90035-003 - Porto Alegre - RS - Brasil e-mail: fernando.araujo@ufrgs.br or adriela.mariath@uol.com.br - Phone: +55-51-3316-5027 /8446-1676 - Fax number: +55-51-3316-5002

Received: February 2, 2007 - Modification: July 23, 2007 - Accepted: September 14, 2007

\begin{abstract}
$T_{\text {he }}$

he purpose of this study was to validate the elastomeric impression after temporary tooth separation as a method of cavitation detection in proximal caries lesions in primary molars with outer half dentin radiolucency. Fifty-one children (4-10 years old), presenting radiolucency in the outer half of the dentin at the proximal surfaces of primary molars and proximal anatomic contact with the adjacent tooth (without restoration/cavitated caries lesion) were enrolled in the study. Temporary tooth separation was performed with an orthodontic rubber ring placed around the contact point during 2-3 days. Thereafter, impression of the proximal surfaces was made. The elastomeric impressions were classified as "non-cavitated" or "cavitated" surfaces. Visual inspection after tooth separation was considered as the gold standard. Examiner reliability of visual inspection after tooth separation was determined (kappa 0.92). Impression examination was repeated every 5 participants to evaluate the reproducibility of the method. The frequency of cavitated lesions was $65 \%$, and $67 \%$ of those were inactive. Sensitivity, specificity, positive and negative predictive values were $0.88 \%$ (95\%CI 0.73-0.95), 0.89\% (95\%CI 0.67-0.97), 0.94\% (95\%CI 0.790.98 ) and $0.80 \%$ (95\%CI 0.58-0.92), respectively. Impression examination showed total agreement regarding cavitation. The evaluation of elastomeric impression after tooth separation is a useful clinical resource in cavitation detection for clinicians and researchers when visual inspection is doubtful.
\end{abstract}

Uniterms: Dental caries; Bitewing radiography; Dentition, primary.

\section{INTRODUCTION}

The diagnosis and treatment of contacting proximal cavitated caries lesions, not detected by conventional clinical examination, have been the subject of several studies ${ }^{6,13,14,23,25}$. Due to its detection complexity, the radiographic image has assumed an important role in the proximal caries lesions diagnosis, making possible to identify up to six times more proximal lesions than the clinical exam alone ${ }^{22}$.

Because of the clinical restraint to proximal cavitation diagnosis, many studies try to associate the presence of the cavitation to radiolucency depth, in order to predict the presence of cavities. In this respect, it is observed that the probability of cavitation is higher for lesions with radiographic extension in dentin than in enamel. However, it is considered controversial in lesions involving the dentinoenamel interface. Therefore, caries lesions with intermediate extension, i.e., those extending from the dentinoenamel junction to half dentin, are considered controversial in treatment decision because the presence of cavitation can range between 20 and 95\% 1,4,6,12,16,17,18,22,25.
The temporary tooth separation technique has been proposed as an alternative diagnostic resource to overcome the difficulty of visually examining proximal surfaces. Some studies compared the direct visual inspection after temporary tooth separation to conventional visual inspection and radiographic analysis and revealed higher accuracy values for direct visual inspection after temporary tooth separation ${ }^{15,21}$. Araújo et al. ${ }^{4}$, evaluated the accuracy of visual inspection after the tooth separation compared to bitewing radiographs. Analyzing 168 high-school students' radiographs, it was observed that $51 \%$ of the radiographically identified lesions were not detected by clinical examination. The authors concluded that direct visual examination after tooth separation is an effective method to confirm the radiographic diagnosis at the proximal surface of permanent teeth, mainly for incipient caries lesions. Findings in primary teeth showed 30\% more identified lesions with direct visualization after tooth separation technique when compared to conventional visual inspection ${ }^{24}$. The authors also observed that the tooth surface influences the clinical diagnosis accuracy, either with or without tooth 
separation, as primary molars mesial surfaces have shown a higher proportion of detected lesions than the distal surfaces. It is still important to stress that pain symptomatology has not been referred in $80 \%$ of the children and, among those who referred some degree of discomfort, only low and intermediate pain scores were recorded ${ }^{24}$.

Proximal surface impression evaluation can be an important resource for detecting the presence of cavitation when chromatic changes, small available visual gap in visualizing and probing raise difficulties in the caries cavitation detection ${ }^{21}$. No study has validated this diagnostic method, although it has been reported as method to detect cavitation ${ }^{15,21,24}$. This diagnostic method can be useful both in the clinical follow-up of proximal caries lesions and in clinical studies that investigate the progression of proximal caries lesions, as the clinical diagnostic of caries progression is difficult to performed.

Therefore, the purpose of this study was to validate the elastomeric impression after tooth separation as a method to detect cavitation in proximal caries lesion in primary molars with outer half dentin radiolucency.

\section{MATERIALAND METHODS}

\section{Sample}

The study was conducted with 51 children, 4-10 years of age. These individuals were in the beginning of their dental treatment in the Pediatric Dental Clinic at the Federal University of Rio Grande do Sul, Brazil and were selected during the year of 2002. The inclusion criteria were: radiolucency in the outer half of the dentin in a primary molar proximal surface; presence of proximal anatomic contact with the adjacent tooth, in this case showing a contact surface without restoration or cavitated caries lesion detected through visual inspection. In cases having with more than one caries lesion fulfilling the inclusion criteria, only one lesion/subject was selected at random. A primary molar was excluded if it presented with another caries lesion, restoration or hypocalcification communicating with the proximal caries lesion selected for the study.

This research was conducted in accordance with the Declaration of Helsinki and was approved by the Ethics in Research Committee of the School of Dentistry (approval number 0036.0.165.000-06, April 2005). The patients were included in this study after patient and parental acceptance and confirmation by signing an informed consent form.

\section{Radiographs and Temporary Tooth Separation}

Bitewing radiographs were taken using number 1 size Ektaspeed Plus films (Eastmann Kodak, USA) in an interproximal device holder. A Gnatus X-ray machine (SP, Brazil), operated at $65 \mathrm{Kvp}, 7.5 \mathrm{~mA}$ and 0.6 seconds, was used to expose the films with the beam aimed perpendicular to the teeth. The exposed films were processed in an automatic processing machine at $27^{\circ} \mathrm{C}$ with a 4.5 -minute processing cycle. The radiographs were examined blindly in random order in a dark frame with a x2 magnification by the examiner using depth scale (D0: sound surface; D1: enamel radiolucency; D2: enamel-dentin junction radiolucency; D3: outer half dentin radiolucency; D4: inner half dentin radiolucency).

Temporary tooth separation was performed using an orthodontic rubber ring for 2 to 3 days, making feasible the proximal surface impression. After the rubber ring was removed, the proximal surface was cleaned (with rotatory brush and dental floss) and a double impression technique was performed with cured addition silicone impression material (Express-3M, São Paulo, SP, Brazil). The impression was evaluated all together, at a second moment, after codification of each impression and without magnification. The surfaces were classified as follows: "non-cavitated" (smooth surface or light roughness on the proximal impression site) or "cavitated" (a collection of positive material on the proximal impression site, indicating a well-defined cavitation).

The visual examination was recorded under relative saliva isolation (with a cotton roll) with the aid of an explorer probe and a flat dental mirror. The probe was used for tactile evaluation of the surface without any pressure. Caries lesion activity was determined according to Figure 1. For the validation study, the findings of visual examination after the temporary tooth separation was the gold standard, thus the active and inactive cavitated lesions corresponded to "cavitated" and the active and inactive non-cavitated caries lesions on visual inspection were the "non-cavitated" on the impression.

The parents and the children were questioned about the occurrence of any symptom during the period of wearing of

\begin{tabular}{|l|l|}
\hline Diagnosis & Clinical characteristics \\
\hline \multirow{2}{*}{ Sound } & Normal enamel translucency and texture \\
Active white spot lesion & Enamel surface is whitish, opaque with bright loss and rough. \\
Inactive white spot lesion & Enamel surface is whitish/brownish, shiny and roughness is present. \\
& Cavitation border: Whitish, rough and opaque enamel surface. \\
Inactive cavitated lesion & Broken enamel surface. Wet, soft and yellowish dentin when exposed. \\
& Cavitation border: Whitish/brownish, smooth and shiny enamel surface. \\
& Broken enamel surface. Dry, hard and brownish dentin when exposed. \\
\hline
\end{tabular}

Adapted from Thylstrup and Fejerskov, 1995; Nyvad, Machiulskiene, Baelum, 1999.

FIGURE 1- Clinical criteria for visual inspection diagnosis 
the orthodontic elastic.

\section{Examiner reproducibility}

The reliability of the examiner was evaluated repeating after a one-week interval the radiographic analysis of 55 bitewing radiographs (kappa 0.84). Reproducibility of visual inspection (kappa 0.74) was performed by re-examining (one-week interval) 60 extracted teeth with and without proximal caries lesion, positioned side-by-side with a millimeter space between the primary molars. The impressions were analyzed twice (classifying in "cavitated” or "non-cavitated” surface), also with a one-week interval, and the reliability was performed.

\section{Data Analysis}

For the validation study, sensitivity, specificity, positive and negative predictive values and their respective 95\% confidence intervals (95\%CI) were calculated considering the visual inspection after temporary separation as the gold standard. Statistical analysis was conducted with SPSS 13(SPSS Inc., Chicago, IL, USA). The individual was the analytical unit and the significance level was set at 5\%.

\section{RESULTS}

The characteristics of the study sample are given in Table 1. Most proximal caries lesions with outer half dentin
TABLE 1- Gender, age, dmf-s, DMF-S and percentage of cavitated lesions in the sample

\section{Variable}

Gender (male/female\%)

$22 / 29(43 / 57 \%)$

dmf-s*

$10.14 \pm 7.81$

DMF-S*

$2.27 \pm 2.80$

Cavitation on visual

inspection examination (\%)

*mean \pm standard deviation.

TABLE 3- Sensitivity, specificity, positive and negative predictive values of the impression

$95 \% \mathrm{Cl}$

\begin{tabular}{lll}
\hline Sensitivity & 0.88 & $0.73-0.95$ \\
Specificity & 0.89 & $0.67-0.97$ \\
Positive predict value & 0.94 & $0.79-0.98$ \\
Negative predict value & 0.80 & $0.58-0.92$ \\
\hline
\end{tabular}

TABLE 2- Distribution of cavitated and non-cavitated lesions on impression and visual inspection examination (goldstandard)

\begin{tabular}{lccc}
\hline & \multicolumn{1}{c}{$\begin{array}{c}\text { Direct Visual Inspection - GOLD STARDARD } \\
\text { Impression } \\
\text { Non-cavitated } \\
\text { (active - inactive) }\end{array}$} & $\begin{array}{c}\text { Cavitated } \\
\text { (active - inactive) }\end{array}$ \\
\hline Non-cavitated & $16(2-14)$ & $4(1-3)$ & $20(39)$ \\
Cavitated & $2(0-2)$ & $29(10-19)$ & $31(61)$ \\
Total $(\%)$ & $18(35)$ & $33(65)$ & 51 \\
\hline
\end{tabular}

radiolucency showed cavitation after tooth separation (gold standard evaluation).

Only 4 out of the 51 children complained about discomfort during use of the rubber ring separators and in only one case an analgesic was administered. Impression reliability analysis showed total agreement. Cavitation findings on impression and visual inspection examination (gold-standard) are presented in Table 2. The distribution of the diagnostic errors is also presented in Table 2. Most caries lesions were clinically diagnosed as inactive lesions. The false positive predictive values occurred on the distal surface of the first primary molars and the false positive values on three distal surface of a primary first molar and one mesial surface of a primary second molar (not in the table).

Sensitivity, specificity, positive and negative predictive values are shown in Table 3. Impression sensitivity and specificity were fairly similar and satisfactory. The modest negative predictive value was a result of significant number of false negatives (Table 2). Its interpretation presumes that in the case of "non-cavitated" lesion on impression, the probability of a non-cavitated lesion is $80 \%$. The relatively high positive predictive values can assure that in the event of identification in the proximal surface impression, the possibility of a real surface rupture is $94 \%$

\section{DISCUSSION}

The present study selected a convenience sample. This can be justified considering the difficulty to compose of study population with children presenting this hard-to-detect caries lesions, not seen in visual inspection but only radiographically 
Nevertheless, the subjects presented different periodontal and caries disease status, all of them in initial treatment at the Pediatric Dentistry Clinic, similar to previous studies ${ }^{1,11,17,23}$. The caries level of this sample is very high compared to the findings of other Brazilian surveys ${ }^{8,10}$. However, observing caries prevalence in permanent teeth, there was comparable data found in similar age groups among the Brazilian southern population $^{5}$. Caries prevalence is important to characterize the sample, as it is expected more caries severity (cavitated lesions and/or lesion progression) in high-risk caries groups ${ }^{26}$.

It is important to stress that not all lesions with such radiolucency have been selected. This work only studied caries lesions with radiolucency of the outer half dentin, which did not show signs of cavitation, such as contact point rupture or extension to free surfaces. In the presence of cavitated lesion with a rupture on the contact point, other factors like loss of the dental arch perimeter ${ }^{9}$ and periodontal involvement ${ }^{7}$ would justify invasive treatment.

An important issue of this study is the fact that $65 \%$ of the caries lesions of the sample presented cavitation after temporary teeth separation. Note that all these lesions had no clinical sign of proximal caries before the separation. The lesions were detected only radiographically. This finding present two important features. First, the radiographic ability in detecting proximal caries, corroborating previous study with low-caries prevalence population in which $33 \%$ of 5 year-old children benefited from bitewing radiographic examination, but only $12 \%$ had dentin caries lesions ${ }^{2}$. The second issue is the expressive number of undetected dentin caries lesion with visual/ tactile clinical examination. This finding is consistent with a representative 5-year-old Swedish children sample ${ }^{2}$, in which $33 \%$ had at least one proximal enamel or dentin caries lesion that was not detected without bitewing examination.

Direct visual inspection was the validation method used to identify cavitated caries in the present study. The use of direct visual examination as a gold standard has been questioned because of its low reproducibility ${ }^{15}$. Additionally, the small gap available for visualization, enamel staining, surface under evaluation and tooth position act as a negative feature on direct visual inspection ${ }^{21,28}$. The size of the cavity is also a matter of discussion. Kielbassa, et al. ${ }^{16}$ evaluated the cavitation rate of proximal caries using magnification aids and observed significantly more cases than previously reported. Nevertheless, clinical examination is a feasible diagnostic method available to clinicians and it is the most widely used method for evaluation in different clinical studies and the only way to diagnose the nature of the lesions, that is, whether they are active or inactive

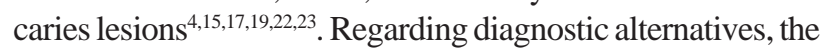
gold standard is defined as the best available method, offering accuracy, reproducibility, feasibility and a justifiable cost-benefit interrelation ${ }^{27}$.

The addition silicone elastomeric material was selected due to its excellent properties, such as high reproduction capacity, dimensional stability, easy usage, good acceptance and noninvasiveness ${ }^{19}$, and also because this material has been used in similar investigatons ${ }^{17,19,23}$. For the present proposal, this material showed a high reliability, as all the repeated impressions agreed in cavitation diagnosis. Analyzing the diagnostic errors (false positive and negative predictive values), it was possible to observe that most of them occurred on the distal surface and in only one case on the mesial surface, corroborating the findings of Rimmer and Pitts ${ }^{24}$, which have shown the greater error possibility in distal surfaces evaluations.

In permanent teeth, most studies evaluating clinical aspects of proximal caries lesions in relation to radiolucency depth showed greater probability of cavitation when dentin radiolucency is present ${ }^{1,6,17,18}$. This identical association in primary teeth reveals outcomes varying from $28 \% \%^{22}, 65 \%{ }^{20}$ and $90 \%{ }^{3,12}$. However, it must be pointed out that some are in vitro studies ${ }^{12,20}$ performed with extracted teeth, which provides weak scientific evidence. In the present study, 33 (65\%) out of 51 proximal caries lesions presented cavitated caries lesion (Table 2), but it is not possible to generalize to all proximal caries lesions with outer half dentin radiolucency, as only lesions with greater diagnosis complexity were included in this study (i.e., those detected only radiographically). Feldens, et al. (2003) ${ }^{12}$ showed that $90 \%$ of the outer half dentin lesions presented cavitation, although this finding is based on 13 proximal surfaces with outer half dentin radiolucency (in a total of 125 caries lesions examined). The same limitation occurred in another in $v$ vitro $^{20}$ study that evaluated the clinical aspect of primary proximal caries lesions. Both studies used extracted teeth and the power of this evidence is very limited.

The comparison of the clinical and radiographic aspects among distinctive populations must take into consideration the different caries experience. In low-caries prevalence groups, it is expected that caries lesion progression (cavitation) occurrs in a smaller proportion compared to high-caries prevalent groups. In the present study, individuals were distinguished by a high caries expression. Therefore, it was expected that most evaluated proximal lesions would reveal cavitation (Table 2). Comparing these data to those of Feldens, et al. ${ }^{12}$, cavitation was not that frequent, although their study did not mention the clinical aspects of caries lesions, whether there were broken marginal crest lesions or caries lesions communicating with smooth surfaces. Data shown in this respect are fairly different and it seems necessary to reach for representative data at each appropriate population. As a result of the analysis of evidence, there is a great difficulty in taking a clinical decision based only on the radiographic examination.

Impression after temporary separation revealed high and satisfactory sensitivity and specificity for a diagnostic method ${ }^{27}$. The positive predictive value that indicates the chance of cavitation when there is cavitation on impression was fairly high, proving that this is a useful method for proximal cavitation evaluation. Although the accuracy parameters (sensitivity, specificity, positive and negative predictive values) have been fairly satisfactory, some authors ${ }^{15,18,21,28}$ state that it is not possible to consider the post-separation visual inspection as a caries cavitation diagnostic validation method, due to the number of false positive and negative values. These negative results actually occurred (Table 3) but the unfeasibility of considering the direct visual inspection as a gold standard is questionable because there is no diagnosis that assures 100\% of accuracy and these data bring safety to be clinically used. 


\section{CONCLUSIONS}

Based on the experimental design of the present investigation, it was concluded that elastomeric impression analysis after temporary separation of primary teeth is a useful cavitation diagnostic resource allowing its use in research and clinical practice when visual inspection is doubtful.

\section{ACKNOWLEDGMENTS}

This work was based on the thesis presented by the first author to the Postgraduate Program of the School of Dentistry of the Federal University of Rio Grande do Sul, Brazil, in partial fulfillment of the requirements for granting the Master's Degree in Dentistry (Pediatric Dentistry Area). This study was partially supported by CAPES (Coordenação de Aperfeiçoamento de Pessoal de Ensino Superior, Brazilian Ministry of Education).

\section{REFERENCES}

1- Akpata ES, Farid MR, al-Saif K, Roberts EA. Cavitation at radiolucent areas on proximal surfaces of posterior teeth. Caries Res. 1996;30(5):3136.

2- Anderson M, Stecksen-Blicks C, Stenlund H, Ranggard L, Tsilingaridis G, Mejare I. Detection of approximal caries in 5-year-old Swedish children. Caries Res. 2005;39(2):92-9.

3- Araujo FB, Araujo DR, Santos CK, de Souza MA. Diagnosis of approximal caries in primary teeth: radiographic versus clinical examination using tooth separation. Am J Dent. 1996;9(2):54-6.

4- Araujo FB, Rosito DB, Toigo E, Santos CK. Diagnosis of approximal caries: radiographic versus clinical examination using tooth separation. Am J Dent. 1992;5(5):245-8.

5- Barbachan e Silva B, Maltz M. [Prevalence of dental caries, gingivitis, and fluorosis in 12-year-old students from Porto Alegre - RS, Brazil, 1998/1999]. Pesqui Odontol Bras. 2001;15(3):208-14.

6- Bille J, Thylstrup A. Radiographic diagnosis and clinical tissue changes in relation to treatment of approximal carious lesions. Caries Res. $1982 ; 16(1): 1-6$

7- Bimstein E. Frequency of alveolar bone loss adjacent to proximal caries in the primary molars and healing due to restoration of the teeth. Pediatr Dent. 1992;14(1):30-3.

8- Carvalho JC, Mestrinho HD, Bezerra AC, Maltz M. Onset, development and arrest of dental caries in Brazilian pre-school children. Clin Oral Investig. 1998;2(2):96-100.

9- Craig GG, Powell KR, Cooper MH. Caries progression in primary molars: 24-month results from a minimal treatment programme. Community Dent Oral Epidemiol. 1981;9(6):260-5.

10 - Dini EL, Holt RD, Bedi R. Prevalence and severity of caries in 3-12year-old children from three districts with different fluoridation histories in Araraquara, SP, Brazil. Community Dent Health. 1998;15(1):44-8.

11 - Ekstrand KR, Bruun G, Bruun M. Plaque and gingival status as indicators for caries progression on approximal surfaces. Caries Res. 1998;32(1):415 .
12 - Feldens CA, Tovo MF, Kramer PF, Feldens EG, Ferreira SH, Finkler $\mathrm{M}$. An in vitro study of the correlation between clinical and radiographic examinations of proximal carious lesions in primary molars. J Clin Pediatr Dent. 2003;27(2):143-7.

13- Grondahl HG. Radiographic caries diagnosis and treatment decisions. Swed Dent J. 1979;3(4):109-17.

14- Hintze H, Wenzel A, Danielsen B. Behaviour of approximal carious lesions assessed by clinical examination after tooth separation and radiography: a 2.5-year longitudinal study in young adults. Caries Res. 1999;33(6):415-22.

15- Hintze H, Wenzel A, Danielsen B, Nyvad B. Reliability of visual examination, fibre-optic transillumination, and bite-wing radiography, and reproducibility of direct visual examination following tooth separation for the identification of cavitated carious lesions in contacting approximal surfaces. Caries Res. 1998;32(3):204-9.

16- Kielbassa AM, Paris S, Lussi A, Meyer-Lueckel H. Evaluation of cavitations in proximal caries lesions at various magnification levels in vitro. J Dent. 2006;34(10):817-22.

17 - Lunder N, von der Fehr FR. Approximal cavitation related to bitewing image and caries activity in adolescents. Caries Res. 1996;30(2):1437

18- Mejare I, Malmgren B. Clinical and radiographic appearance of proximal carious lesions at the time of operative treatment in young permanent teeth. Scand J Dent Res. 1986;94(1):19-26.

19- Mialhe FL, Pereira AC, Pardi V, de Castro Meneghim M. Comparison of three methods for detection of carious lesions in proximal surfaces versus direct visual examination after tooth separation. J Clin Pediatr Dent. 2003;28(1):59-62.

20- Nielsen LL, Hoernoe M, Wenzel A. Radiographic detection of cavitation in approximal surfaces of primary teeth using a digital storage phosphor system and conventional film, and the relationship between cavitation and radiographic lesion depth: an in vitro study. Int J Paediatr Dent. 1996;6(3):167-72.

21 - Pitts NB, Longbottom C. Temporary tooth separation with special reference to the diagnosis and preventive management of equivocal approximal carious lesions. Quintessence Int. 1987;18(8):563-73.

22 - Pitts NB, Rimmer PA. An in vivo comparison of radiographic and directly assessed clinical caries status of posterior approximal surfaces in primary and permanent teeth. Caries Res. 1992;26(2):146-52.

23- Ratledge DK, Kidd EA, Beighton D. A clinical and microbiological study of approximal carious lesions. Part 1: the relationship between cavitation, radiographic lesion depth, the site-specific gingival index and the level of infection of the dentine. Caries Res. 2001;35(1):3-7.

24- Rimmer PA, Pitts NB. Temporary elective tooth separation as a diagnostic aid in general dental practice. Br Dent J. 1990;169(3-4):8792.

25. Rugg-Gunn AJ. Approximal carious lesions. A comparison of the radiological and clinical appearances. Br Dent J. 1972;133(11):481-4.

26- Vanderas AP, Gizani S, Papagiannoulis L. Progression of proximal caries in children with different caries indices: a 4-year radiographic study. Eur Arch Paediatr Dent. 2006;7(3):148-52.

27- Verdonschot EH, Angmar-Mansson B, ten Bosch JJ, Deery CH, Huysmans MC, Pitts NB, et al. Developments in caries diagnosis and their relationship to treatment decisions and quality of care. ORCA Saturday Afternoon Symposium 1997. Caries Res. 1999;33(1):32-40.

28- Wenzel A. Digital radiography and caries diagnosis. Dentomaxillofac Radiol. 1998;27(1):3-11. 